\title{
Silica-Supported Dichlorophosphate: a Recoverable Cyclodehydrant for the Eco-Friendly Synthesis of 2,5-Disubstituted 1,3,4-Oxadiazoles under Solvent-Free and Microwave Irradiation Conditions
}

\author{
Zheng Li,* Anguo Zhu, Xuerong Mao, Xiunan Sun and Xue Gong \\ Gansu Key Laboratory of Polymer Materials, College of Chemistry and Chemical Engineering, \\ Northwest Normal University, Lanzhou, Gansu, 730070, PR China
}

\begin{abstract}
Uma série de compostos simétricos e assimétricos de 2,5-dissubstituídos 1,3,4-oxadiazol foram sintetizados eficientemente a partir do ciclodesidratação das diacilhidrazinas, usando a sílica suportada com o diclorofosfato, como recuperador do ciclodesidratação sem solvente e sob irradiação por microondas. Este processo tem vantagens de ser não corrosivo e nem poluente, apresenta ainda velocidade acelerada, alto rendimento e simples procedimento experimental.
\end{abstract}

A series of symmetrical and unsymmetrical 2,5-disubstituted 1,3,4-oxadiazoles were efficiently synthesized from the cyclodehydration of diacylhydrazines by using silica-supported dichlorophosphate as a recoverable cyclodehydrant in solvent-free medium under microwave irradiation. This protocol has advantages of no corrosion, no environmental pollution, accelerated rate, high yield and simple work-up procedure.

Keywords: silica-supported dichlorophosphate, diacylhydrazine, 1,3,4-oxadiazole, microwave irradiation, solvent-free synthesis

\section{Introduction}

2,5-Disubstituted 1,3,4-oxadiazoles are a class of heterocycles which have a wide range of biological activities in medicinal chemistry including antimicrobial, ${ }^{1}$ anti-inflammatory, ${ }^{2}$ anticonvulsant, ${ }^{3}$ anti-HIV, ${ }^{4}$ and antituberculostatic ${ }^{5}$ activities. They have also been used in pesticide chemistry, ${ }^{6}$ polymer ${ }^{7}$ and material science. ${ }^{8-10}$ 1,3,4-Oxadiazoles have been synthesized by traditional methodology via several approaches, three of the more popular being the cyclization of diacylhydrazines, ${ }^{11}$ the cyclization of acylthiosemicarbazides, ${ }^{12}$ and the oxidation of acylhydrozones. ${ }^{13,14}$ However, long reaction procedure, extended reaction period at elevated temperature, and harsh reagents, e.g., $\mathrm{BF}_{3}-\mathrm{OEt}_{2},{ }^{15}$ 1,1,1,3,3,3-hexamethydisilazane, ${ }^{16}$ triflic anhydride, ${ }^{17}$ phosphorus pentoxide,${ }^{18}$ polyphosphoric acid, ${ }^{19}$ thionyl chloride, ${ }^{20}$ phosphorus oxychloride, ${ }^{21}$ and sulfuric acid ${ }^{22}$ are usually encountered. These liquid reagents not only make the reaction systems corrosive, but also cause the severe environmental problems because of their difficult separation and recovery after reactions. Recently, chemists reported some greener approaches

*e-mail: lizheng@nwnu.edu.cn using polymer-supported Burgess reagent, ${ }^{23-24}$ silica sulfuric acid, ${ }^{25}$ and solid supported Nafion NR50 ${ }^{26}$ as dehydrants to the synthesis of 2,5-disubstituted 1,3,4-oxadiazoles, which could efficiently avoid the corrosion and pollution caused by using other liquid homogeneous reagents because of its easy separation.

The development of solvent-free organic synthesis is of current interest because of the many advantages, such as reduced pollution, low cost, simplicity in process and handling, potential applications in combinatorial chemistry and chemical industry. ${ }^{27-29}$

Microwave energy has also developed into a useful technique for a variety of applications in organic synthesis, ${ }^{30-32}$ especially for the solvent-free reactions ${ }^{33,34}$ since the solvent-free MW-assisted reactions can provide an opportunity to work with open vessels thus avoiding the risk of high pressure development and increasing the potential of such reaction to upscale, and has advantage of rapid reaction rate, high yield and simple work-up procedure.

In this paper, we describe an eco-friendly new method that utilizes silica-supported dichlorophosphate as a recoverable cyclodehydrant for the synthesis of 2,5-disubstituted 1,3,4-oxadiazoles from 1,2-diacylhydrazines under solventfree and microwave irradiation condition. 


\section{Experimental}

IR spectra were recorded using $\mathrm{KBr}$ pellets on a Digilab FTS 3000 FTIR spectrophotometer and ${ }^{1} \mathrm{H}$ NMR spectra on a Mercury Plus-400 instrument using $\mathrm{CDCl}_{3}$ as solvent and $\mathrm{Me}_{4} \mathrm{Si}$ as internal standard. Elemental analyses were performed on a Vario El Elemental Analysis instrument. Melting points were observed in an electrothermal melting point apparatus, and the values are uncorrected. Microwave reactions were conducted in a modified microwave oven fitted with a condenser (LG-WP650, $2450 \mathrm{MHz}$, China). BET surface area of silica-supported dichlorophosphate was determined from $\mathrm{N}_{2}$ adsorption-desorption isotherms using a Surface Area Analyzer (BET, Quantachrome Instruments Nova 2000 series, USA). 1,2-Diacylhydrazines were prepared according to literature procedure. ${ }^{15}$

\section{Preparation of silica-supported dichlorophosphate}

The suspension of silica gel (10 g, 80-200 mesh) in phosphorus oxychloride $(50 \mathrm{~mL})$ was refluxed for $48 \mathrm{~h}$. The released $\mathrm{HCl}$ gas was absorbed by dilute aqueous solution of sodium hydroxide. Then the mixture was filtered in a drybox, washed with dry dichloromethane $(6 \times 10 \mathrm{~mL})$ and dried in vacuum to give $13 \mathrm{~g}$ of product. IR $(\mathrm{KBr}): 571\left(v_{\mathrm{P}-\mathrm{Cl}}\right)$. The silica supporting capacity for $\mathrm{POCl}_{2}$ is $c a .1 .92 \mathrm{mmol} \mathrm{g}^{-1}$ (which was calculated according to weight increase of silica). The specific surface area for the sample is $c a .82 \mathrm{~m}^{2} \mathrm{~g}^{-1}$.

General procedure for preparation of 2,5-disubstituted 1,3,4-oxadiazoles

The mixture of 1,2-diacylhydrazine $(0.7 \mathrm{mmol})$ and silica-supported dichlorophosphate $(0.52 \mathrm{~g}, 1.0 \mathrm{mmol}$ based on supported $\mathrm{POCl}_{2}$ ) was ground in a mortar with a pestle at room temperature without any solvents for $15 \mathrm{~min}$. Then the mixture was transferred into a beaker, and irradiated in a microwave oven for $2 \mathrm{~min}$ at $455 \mathrm{~W}$ of microwave power. Then the resulting mixture was extracted with $10 \mathrm{~mL}$ of acetone to recover the used silica-supported reagent, and the extract was evaporated off the solvent to give the crude product, which was further purified by column chromatography to afford pure product. The analytical data for represent compounds are shown below.

2,5-Di(3-chlorophenyl)-1,3,4-oxadiazole (2c): White solid; IR (KBr) $v_{\max } / \mathrm{cm}^{-1}: 3070,1542,1262,1084 ;{ }^{1} \mathrm{H} \mathrm{NMR}$ $\left(400 \mathrm{MHz}, \mathrm{CDCl}_{3}\right): \delta 8.13(\mathrm{~s}, 2 \mathrm{H}), 8.05(\mathrm{~d}, J 7.6 \mathrm{~Hz}, 2 \mathrm{H})$, 7.48-7.56 (m, 4H); Anal. Calcd. for $\mathrm{C}_{14} \mathrm{H}_{8} \mathrm{Cl}_{2} \mathrm{~N}_{2} \mathrm{O}: \mathrm{C}, 57.76$; H, 2,77; N, 9.62. Found: C, 57.82; H, 2.78; N, 9.64.

2-(4-Methoxyphenyl)-5-phenyl-1,3,4-oxadiazole (2n): White solid; IR (KBr) $v_{\max } / \mathrm{cm}^{-1}: 3066,2924,1613,1499$,
1263, 1016; ${ }^{1} \mathrm{H}$ NMR (400 MHz, $\mathrm{CDCl}_{3}$ ): $\delta$ 8.06-8.07 $(\mathrm{m}, 2 \mathrm{H}), 8.02(\mathrm{~d}, J 8.8 \mathrm{~Hz}, 2 \mathrm{H}), 7.46-7.48(\mathrm{~m}, 3 \mathrm{H}), 6.97$ $(\mathrm{d}, J 8.8 \mathrm{~Hz}, 2 \mathrm{H}), 3.83(\mathrm{~s}, 3 \mathrm{H})$; Anal. Calcd. for $\mathrm{C}_{15} \mathrm{H}_{12} \mathrm{~N}_{2} \mathrm{O}_{2}$ : C, 71.42; H, 4.79; N, 11.10. Found: C, 71.33; H, 4.80; N, 11.13 .

2-(3-Methylphenyl)-5-(2-chlorophenyl)-1,3,4oxadiazole (2p): White solid; IR (KBr) $v_{\max } / \mathrm{cm}^{-1}: 3054$, 2933, 1608, 1501, 1264, 1018; ${ }^{1} \mathrm{H}$ NMR (400 MHz, $\left.\mathrm{CDCl}_{3}\right): \delta 6.99-8.03(\mathrm{~m}, 8 \mathrm{H}), 2.32(\mathrm{~s}, 3 \mathrm{H})$; Anal. Calcd. for $\mathrm{C}_{15} \mathrm{H}_{11} \mathrm{ClN}_{2} \mathrm{O}$ : C, 66.55; H, 4.10; N, 10.35. Found: C, 66.62; H, 4.08; N, 10.31.

2-(3-Methylphenyl)-5-(3-chlorophenyl)-1,3,4oxadiazole (2q): White solid; IR (KBr) $v_{\max } / \mathrm{cm}^{-1}: 3052$, 2931, 1603, 1504, 1265, 1014; ${ }^{1} \mathrm{H}$ NMR (400 MHz, $\left.\mathrm{CDCl}_{3}\right): \delta$ 7.02-8.01 (m, 8H), $2.31(\mathrm{~s}, 3 \mathrm{H})$; Anal. Calcd. for $\mathrm{C}_{15} \mathrm{H}_{11} \mathrm{ClN}_{2} \mathrm{O}$ : C, 66.55; H, 4.10; N, 10.35. Found: C, 66.49; H, 4.12; N, 10.32.

2-(3-Methylphenyl)-5-(4-chlorophenyl)-1,3,4oxadiazole (2r): White solid; IR ( $\mathrm{KBr}) v_{\max } / \mathrm{cm}^{-1}: 3044$, 2928, 1610, 1496, 1260, 1012; ${ }^{1} \mathrm{H}$ NMR (400 MHz, $\left.\mathrm{CDCl}_{3}\right): \delta 6.96-8.00(\mathrm{~m}, 8 \mathrm{H}), 2.31(\mathrm{~s}, 3 \mathrm{H})$; Anal. Calcd. for $\mathrm{C}_{15} \mathrm{H}_{11} \mathrm{ClN}_{2} \mathrm{O}$ : C, 66.55; H, 4.10; N, 10.35. Found: C, 66.59; H, 4.13; N, 10.29.

2-(3-Methylphenyl)-5-(4-methoxyphenyl)-1,3,4oxadiazole (2s): White solid; IR (KBr) $v_{\max } / \mathrm{cm}^{-1}: 3032$, 2921, 1603, 1504, 1273, 1012; ${ }^{1} \mathrm{H} \mathrm{NMR}$ (400 MHz, $\mathrm{CDCl}_{3}$ ): $\delta$ 6.91-7.95 (m, 8H), $3.82(\mathrm{~s}, 3 \mathrm{H}), 2.30(\mathrm{~s}, 3 \mathrm{H})$; Anal. Calcd. for $\mathrm{C}_{16} \mathrm{H}_{14} \mathrm{~N}_{2} \mathrm{O}_{2}: \mathrm{C}, 72.16 ; \mathrm{H}, 5.30 ; \mathrm{N}, 10.52$. Found: C, 72.19; H, 5.28; N, 10.57.

2-(3-Methylphenyl)-5-(3-nitrophenyl)-1,3,4-oxadiazole (2t): Yellow solid; IR (KBr) $v_{\max } / \mathrm{cm}^{-1}: 3071,2945$, 1615, 1509, 1269, 1025; ${ }^{1} \mathrm{H}$ NMR (400 MHz, $\mathrm{CDCl}_{3}$ ): d 7.11-8.25(m, 8H), 2.33 (s, 3H); Anal. Calcd. for $\mathrm{C}_{15} \mathrm{H}_{11} \mathrm{~N}_{3} \mathrm{O}_{3}$ : C, 64.05; H, 3.94; N, 14.94. Found: C, 64.11; H, 3.96; N, 14.88 .

2-Phenyl-5-heptyl-1,3,4-oxadiazole (2u): Oil; IR (KBr) $v_{\max } / \mathrm{cm}^{-1}: 2950,2921,1566,1082 ;{ }^{1} \mathrm{H}$ NMR (400 MHz, $\mathrm{CDCl}_{3}$ ): $\delta 7.36-7.90(\mathrm{~m}, 5 \mathrm{H}), 2.94(\mathrm{~m}, 2 \mathrm{H}), 1.84$ (m, 2H), $1.54(\mathrm{~m}, 2 \mathrm{H}), 1.43(\mathrm{~m}, 2 \mathrm{H}), 1.32(\mathrm{~m}, 4 \mathrm{H}), 0.88$ (t, J 6.4 Hz, 3H); Anal. Calcd. for $\mathrm{C}_{15} \mathrm{H}_{20} \mathrm{~N}_{2} \mathrm{O}: \mathrm{C}, 73.74 ; \mathrm{H}$, 8.25; N, 11.47. Found: C, 73.67; H, 8.27; N, 11.51.

2-(3-Methylphenyl)-5-heptyl-1,3,4-oxadiazole (2v): Oil; IR (KBr) $v_{\max } / \mathrm{cm}^{-1}: 2943,2919,1566,1085$; ${ }^{1} \mathrm{H}$ NMR (400 MHz, $\mathrm{CDCl}_{3}$ ): $\delta$ 7.33-7.88 (m, 4H), 2.92 $(\mathrm{m}, 2 \mathrm{H}), 2.32(\mathrm{~s}, 3 \mathrm{H}), 1.82(\mathrm{~m}, 2 \mathrm{H}), 1.51(\mathrm{~m}, 2 \mathrm{H}), 1.42$ (m, 2H), 1.30 (m, 4H), 0.87 (t, J 6.4 Hz, 3H); Anal. Calcd. for $\mathrm{C}_{16} \mathrm{H}_{22} \mathrm{~N}_{2} \mathrm{O}$ : C, 74.38; H, 8.58; N, 10.84. Found: C, 74.46; H, 8.55; N, 10.79 .

2-(4-Chlorophenyl)-5-heptyl-1,3,4-oxadiazole (2w): White solid; IR (KBr) $v_{\max } / \mathrm{cm}^{-1}: 2953,2924,1579,1091$; ${ }^{1} \mathrm{H}$ NMR (400 MHz, $\mathrm{CDCl}_{3}$ ): $\delta 7.98(\mathrm{~d}, J 8.4 \mathrm{~Hz}, 2 \mathrm{H})$, 

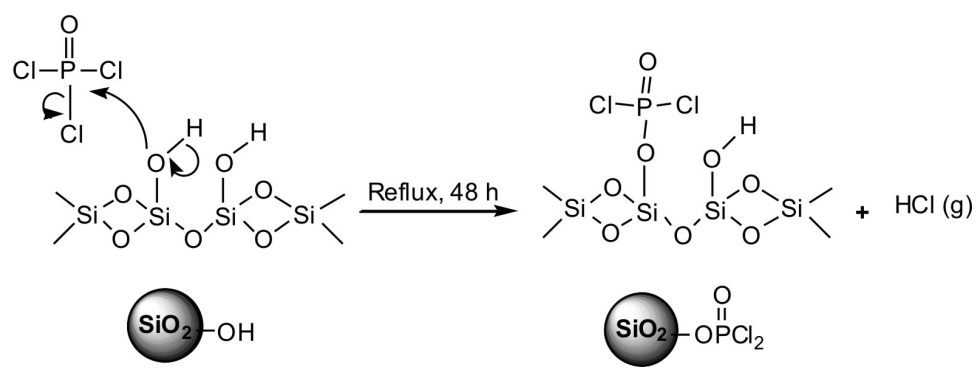

Scheme 1. Synthesis of silica-supported dichlorophosphate.

$7.48(\mathrm{~d}, J 8.4 \mathrm{~Hz}, 2 \mathrm{H}), 2.94(\mathrm{~m}, 2 \mathrm{H}), 1.85(\mathrm{~m}, 2 \mathrm{H})$, $1.56(\mathrm{~m}, 2 \mathrm{H}), 1.44(\mathrm{~m}, 2 \mathrm{H}), 1.33(\mathrm{~m}, 4 \mathrm{H}), 0.90(\mathrm{t}, J 6.8$ $\mathrm{Hz}, 3 \mathrm{H}$ ); Anal. Calcd. for $\mathrm{C}_{15} \mathrm{H}_{19} \mathrm{ClN}_{2} \mathrm{O}: \mathrm{C}, 64.63$; H, 6.87; N, 10.05. Found: C, 64.70; H, 6.86; N, 10.02.

2-(4-Nitrophenyl)-5-heptyl-1,3,4-oxadiazole (2x): Yellow solid; IR (KBr) $v_{\max } / \mathrm{cm}^{-1}: 2963,2933,1586$, 1099; ${ }^{1} \mathrm{H}$ NMR (400 MHz, $\mathrm{CDCl}_{3}$ ): $\delta 8.4(\mathrm{~d}, J 8.4 \mathrm{~Hz}$, 2H), 7.58 (d, J $8.4 \mathrm{~Hz}, 2 \mathrm{H}), 2.96(\mathrm{~m}, 2 \mathrm{H}), 1.86(\mathrm{~m}, 2 \mathrm{H})$, 1.57 (m, 2H), $1.44(\mathrm{~m}, 2 \mathrm{H}), 1.35(\mathrm{~m}, 4 \mathrm{H}), 0.91$ (t, J 6.8 $\mathrm{Hz}, 3 \mathrm{H}$ ); Anal. Calcd. for $\mathrm{C}_{15} \mathrm{H}_{19} \mathrm{~N}_{3} \mathrm{O}_{3}$ : C, 62.27; H, 6.62; N, 14.52. Found: C, 62.23; H, 6.59; N, 14.48.

2-(2-Chlorophenyl)-5-heptyl-1,3,4-oxadiazole (2y): Oil; IR (KBr) $v_{\max } / \mathrm{cm}^{-1}: 2951,2922,1577,1090 ;{ }^{1} \mathrm{H}$ NMR (400 MHz, $\left.\mathrm{CDCl}_{3}\right): \delta$ 7.50-7.97 (m, 4H), $2.93(\mathrm{~m}, 2 \mathrm{H})$, $1.85(\mathrm{~m}, 2 \mathrm{H}), 1.57$ (m, 2H), $1.45(\mathrm{~m}, 2 \mathrm{H}), 1.31$ (m, 4H), 0.90 (t, J 6.8 Hz, 3H); Anal. Calcd. for $\mathrm{C}_{15} \mathrm{H}_{19} \mathrm{ClN}_{2} \mathrm{O}: \mathrm{C}, 64.63$; H, 6.87; N, 10.05. Found: C, 64.57; H, 6.84; N, 10.00.

2-(2-Chlorophenyl)-5-hexyl-1,3,4-oxadiazole (2z): Oil; IR(KBr) $v_{\max } / \mathrm{cm}^{-1}: 3069,2930,1574,1461,1102 ;{ }^{1} \mathrm{H}$ NMR (400 MHz, $\left.\mathrm{CDCl}_{3}\right): \delta 7.97(\mathrm{~m}, 1 \mathrm{H}), 7.38-7.55(\mathrm{~m}, 3 \mathrm{H}), 2.94$ (t, J7.6 Hz, 2H), 1.83-1.90 (m, 2H), 1.32-1.63 (m, 6H), 0.94 (t, J 6.4 Hz, 3H); Anal. Calcd. for $\mathrm{C}_{14} \mathrm{H}_{17} \mathrm{ClN}_{2} \mathrm{O}: \mathrm{C}, 63.51$; H, 6.47; N, 10.58. Found: C, 63.67; H, 6.45; N, 10.60 .

\section{Results and Discussion}

Silica-supported dichlorophosphate was prepared by reaction of silica gel with phosphorus oxychloride under refluxing condition (Scheme 1). The reaction could afford the desired product in excellent yield. Figure 1 shows the IR spectra of silica-supported dichlorophosphate and silica gel. Both IR spectra show the typical broad absorption peaks of Si-O-Si bond at $1103 \mathrm{~cm}^{-1}$. Hydrogen-bonded silanol groups give bands in the region of $3300-3600 \mathrm{~cm}^{-1}$. Especially, a new strong peak at $571 \mathrm{~cm}^{-1}$ is exhibited in the spectrum of silica-supported dichlorophosphate, which is attributable to the absorption of $\mathrm{P}-\mathrm{Cl}$. The specific surface area for the sample is $c a .82 \mathrm{~m}^{2} \mathrm{~g}^{-1}$.

In order to explore the availability of silica supported dichlorophosphate as a recoverable cyclodehydrant under

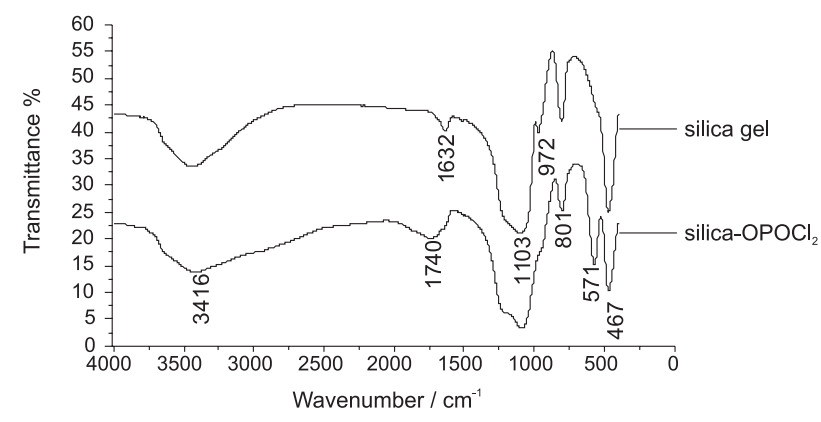

Figure 1. The IR spectra of silica gel and silica-supported dichlorophosphate.

solvent-free and microwave irradiation, the synthesis of 2,5-diphenyl-1,3,4-oxadiazole (Table 1, 2a) from 1,2-dibenzoylhydraine was selected as a model reaction. The optimal reaction conditions including reactant ratio, reaction time, and microwave power were investigated. It was found that when the ratio of 1,2-dibenzoylhydraine to silica-supported dichlorophosphate was 1:1.4 and the microwave power was $455 \mathrm{~W}$ (which was determined by power adjustor of microwave oven), the reaction gave the highest yield within $2 \mathrm{~min}$. Using the similar condition, a series of 2,5-disubstituted-1,3,4-oxadiazoles (Table 1, $\mathbf{2 a - z )}$ were synthesized in high yield. The protocol is suitable for cyclodehydrating synthesis of aryl, alkyl and heterocyclyl substituted 1,3,4-oxadiazoles. Various aryl substituted 1,3,4-oxadiazoles could be easily synthesized by this method. Different substituents (4-MeO, 2-Me, $3-\mathrm{Me}, 2-\mathrm{Cl}, 3-\mathrm{Cl}, 4-\mathrm{Cl}, 3-\mathrm{NO}_{2}$ and $4-\mathrm{NO}_{2}$ ) on the aryl rings were have no significant effect on the reaction conditions and the reaction yield. Alkyl groups, such as hexyl and heptyl, substituted 1,3,4-oxadiazoles could also be readily prepared by this route (Table $1, \mathbf{2 u - z}$ ). In addition, heteroaryl group, such as furyl, substituted 1,3,4-oxadiazoles could also be synthesized from this approach (Table 1, 2f and $\mathbf{2 m}$ ). Meanwhile, this protocol was suitable to the synthesis of both symmetrical (Table 1, 2a-g) and unsymmetrical (Table 1, 2h-z) 1,3,4-oxadiazoles. After the reactions the desired products 
Table 1. Synthesis of 2,5-disubstituted 1,3,4-oxadiazoles by using silica-supported dichlorophosphate as cyclodehydrant

\begin{tabular}{|c|c|c|c|c|}
\hline Compound & $\mathrm{R}^{1}$ & $\mathrm{R}^{2}$ & Yield $/(\%)^{a}$ & $\mathrm{mp} /\left({ }^{\circ} \mathrm{C}\right)$ \\
\hline $2 a$ & $\mathrm{C}_{6} \mathrm{H}_{5}$ & $\mathrm{C}_{6} \mathrm{H}_{5}$ & 80 & $132-134(133-135)^{35}$ \\
\hline $2 b$ & $2-\mathrm{ClC}_{6} \mathrm{H}_{4}$ & $2-\mathrm{ClC}_{6} \mathrm{H}_{4}$ & 88 & $94-96(94-95)^{36}$ \\
\hline $2 \mathrm{c}$ & $3-\mathrm{ClC}_{6} \mathrm{H}_{4}$ & $3-\mathrm{ClC}_{6} \mathrm{H}_{4}$ & 90 & $152-154(154-156)^{15}$ \\
\hline $2 d$ & $2-\mathrm{CH}_{3} \mathrm{C}_{6} \mathrm{H}_{4}$ & $2-\mathrm{CH}_{3} \mathrm{C}_{6} \mathrm{H}_{4}$ & 91 & $123-125(130-131)^{37}$ \\
\hline $2 e$ & $3-\mathrm{CH}_{3} \mathrm{C}_{6} \mathrm{H}_{4}$ & $3-\mathrm{CH}_{3} \mathrm{C}_{6} \mathrm{H}_{4}$ & 95 & $76-78(80)^{38}$ \\
\hline $2 f$ & 2-furyl & 2-furyl & 79 & $136-138(136-138)^{39}$ \\
\hline $2 \mathrm{~g}$ & $4-\mathrm{CH}_{3} \mathrm{OC}_{6} \mathrm{H}_{4}$ & $4-\mathrm{CH}_{3} \mathrm{OC}_{6} \mathrm{H}_{4}$ & 77 & $152-154(155-157)^{39}$ \\
\hline $2 \mathrm{~h}$ & $\mathrm{C}_{6} \mathrm{H}_{5}$ & 2- $\mathrm{CH}_{3} \mathrm{C}_{6} \mathrm{H}_{4}$ & 90 & $94-96(101)^{40}$ \\
\hline $2 \mathbf{i}$ & $\mathrm{C}_{6} \mathrm{H}_{5}$ & $3-\mathrm{CH}_{3} \mathrm{C}_{6} \mathrm{H}_{4}$ & 93 & $98-100(107-109)^{41}$ \\
\hline $2 \mathbf{j}$ & $\mathrm{C}_{6} \mathrm{H}_{5}$ & $2-\mathrm{ClC}_{6} \mathrm{H}_{4}$ & 93 & $96-98(99)^{42}$ \\
\hline $2 k$ & $\mathrm{C}_{6} \mathrm{H}_{5}$ & 3- $\mathrm{ClC}_{6} \mathrm{H}_{4}$ & 95 & $117-119(122-123)^{40}$ \\
\hline 21 & $\mathrm{C}_{6} \mathrm{H}_{5}$ & 4- $\mathrm{ClC}_{6} \mathrm{H}_{4}$ & 80 & $158-160(158-159)^{35}$ \\
\hline $2 \mathrm{~m}$ & $\mathrm{C}_{6} \mathrm{H}_{5}$ & 2-furyl & 88 & $97-99(98-100)^{35}$ \\
\hline $2 n$ & $\mathrm{C}_{6} \mathrm{H}_{5}$ & $4-\mathrm{CH}_{3} \mathrm{OC}_{6} \mathrm{H}_{4}$ & 85 & $146-148(145-146)^{35}$ \\
\hline 20 & $\mathrm{C}_{6} \mathrm{H}_{5}$ & $4-\mathrm{NO}_{2} \mathrm{C}_{6} \mathrm{H}_{4}$ & 90 & $196-198(200-203)^{35}$ \\
\hline $2 p$ & $3-\mathrm{CH}_{3} \mathrm{C}_{6} \mathrm{H}_{4}$ & $2-\mathrm{ClC}_{6} \mathrm{H}_{4}$ & 83 & $86-88$ \\
\hline $2 q$ & $3-\mathrm{CH}_{3} \mathrm{C}_{6} \mathrm{H}_{4}$ & $3-\mathrm{ClC}_{6} \mathrm{H}_{4}$ & 88 & $100-102$ \\
\hline $2 r$ & $3-\mathrm{CH}_{3} \mathrm{C}_{6} \mathrm{H}_{4}$ & 4- $\mathrm{ClC}_{6} \mathrm{H}_{4}$ & 90 & $128-130$ \\
\hline $2 s$ & $3-\mathrm{CH}_{3} \mathrm{C}_{6} \mathrm{H}_{4}$ & $4-\mathrm{CH}_{3} \mathrm{OC}_{6} \mathrm{H}_{4}$ & 95 & $120-122$ \\
\hline $2 \mathrm{t}$ & $3-\mathrm{CH}_{3} \mathrm{C}_{6} \mathrm{H}_{4}$ & $3-\mathrm{NO}_{2} \mathrm{C}_{6} \mathrm{H}_{4}$ & 90 & $126-128$ \\
\hline $2 u$ & $n$-heptyl & $\mathrm{C}_{6} \mathrm{H}_{5}$ & 95 & oil \\
\hline $2 v$ & $n$-heptyl & $3-\mathrm{CH}_{3} \mathrm{C}_{6} \mathrm{H}_{4}$ & 93 & oil \\
\hline $2 w$ & $n$-heptyl & 4- $\mathrm{ClC}_{6} \mathrm{H}_{4}$ & 87 & $62-64$ \\
\hline $2 x$ & $n$-heptyl & $4-\mathrm{NO}_{2} \mathrm{C}_{6} \mathrm{H}_{4}$ & 78 & $54-56$ \\
\hline $2 y$ & $n$-heptyl & $2-\mathrm{ClC}_{6} \mathrm{H}_{4}$ & 80 & oil \\
\hline $2 \mathrm{z}$ & $n$-hexyl & $2-\mathrm{ClC}_{6} \mathrm{H}_{4}$ & 76 & oil \\
\hline
\end{tabular}

${ }^{a}$ Yields refer to the isolated products.

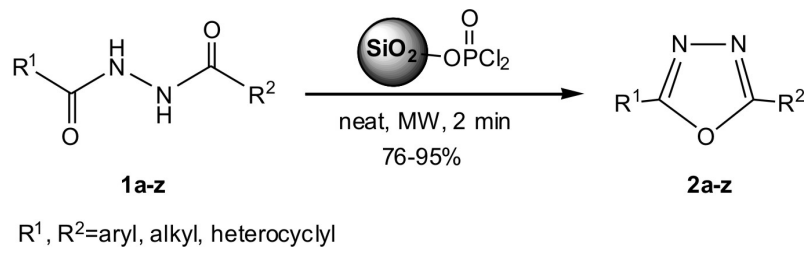

Scheme 2. Synthesis of 2,5-disubstituted 1,3,4-oxadiazoles.

could be easily isolated from the reaction systems only by extraction, and the used silica-supported cyclodehydrant could be recovered simply by filtration, and this efficiently avoided the corrosion and pollution caused by using liquid dehydrant, such as phosphorus oxychloride. The pure products could be readily obtained by evaporating off the solvent and purified by column chromatography.
In contrast, the selected reaction for $\mathbf{2 a}$ was also conducted by using oil-bath heating at the same temperature as the microwave method $\left(140^{\circ} \mathrm{C}\right)$, but no any product was observed after 2 min of reaction.

\section{Conclusion}

Silica-supported dichlorophosphate was found to be an efficient cyclodehydrant for the synthesis of 2,5-disubstituted 1,3,4-oxadiazoles from 1,2-diacylhydrazines in solvent-free medium under microwave irradiation. This protocol is suitable to the synthesis of alkyl, aryl and heterocyclyl substituted symmetrical and unsymmetrical 1,3,4-oxadiazoles, and has advantages of no corrosion, no environmental pollution, accelerated rate, high yield and simple work-up procedure. 


\section{Acknowledgements}

The authors thank the National Natural Science Foundation of China (20772096) and Gansu Natural Science Foundation (3ZS061-A25-033) for the financial support of this work.

\section{References}

1. Ates, O.; Kocabalkanli, A.; Cesur, N.; Otuk, G.; Il Farmaco 1998, 53, 541.

2. Amir, M.; Kumar, S.; Indian J. Heterocycl. Chem. 2004, 14, 51.

3. Almasirad, A.; Tabatabai, S. A.; Faizi, M.; Kebriaeezadeh, A.; Mehrabi, N.; Dalvandia, A.; Shafiee, A.; Bioorg. Med. Chem. Lett. 2004, 14, 6057.

4. Zareef, M.; Iqbal, R.; Al-Masoudi, N. A.; Zaidi, J. H.; Arfan, M.; Heteroat. Chem. 2007, 18, 425.

5. Yar, M. S.; Siddiqui, A. A.; Ali, M. A.; J. Chin. Chem. Soc. 2007, 54, 5.

6. Mohan, T. P.; Vishalakshi, B.; Bhat, K. S.; Rao, K. S.; Kendappa, G. N.; Indian J. Chem., Sect. B, 2004, 43, 1798.

7. Schulz, B.; Kaminorz, Y.; Brehmer, L.; Synth. Met. 1997, 84, 449.

8. Mormile, P.; Petti, L.; Gillo, M.; Laurienzo, P.; Malinconico, M.; Roviello, A.; Lipson, S.; Blau, W. J.; Mater. Chem. Phys. 2002, 77, 945.

9. Cristiano, R.; Vieira, A. A.; Ely, F.; Gallardo, H.; Liq. Cryst. 2006, 33, 381.

10. Cristiano, R.; Oliveira Santos, D. M. P.; Gallardo, H.; Liq. Cryst. 2005, 32, 7 .

11. Mashraqui, S. H.; Ghadigaonkar, S. G.; Kenny, R. S.; Synth. Commun. 2003, 33, 2541.

12. Wang, X. C.; Li, Z.; Wei, B. G.; Yang, J. Y. Synth. Commun. 2002, 32, 1097.

13. Dabiri, M.; Salehi, P.; Baghbanzadeh, M.; Bahramnejad, M.; Tetrahedron Lett. 2006, 47, 6983.

14. Rostamizadeh, S.; Housaini, S. A. G.; Tetrahedron Lett. 2004, 45, 8753.

15. Tandon, V. K.; Chhor, R. B.; Synth. Commun. 2001, 31, 1727.

16. Diana, G. D.; Volkots, D. L.; Nitz, T. J.; Biailly, T. R.; Long, M. A.; Vesico, N.; Aldous, A.; Pevear, D. C.; Dukto, F. J.; J. Med. Chem. 1994, 37, 2421.

17. Liras, S.; Allen, M. P.; Segelstein, B. E.; Synth. Commun. 2000, 30, 437.

18. Carlsen, H. J.; Jorgensen, K. B.; J. Heterocycl. Chem. 1994, $31,805$.

19. Tully, W. R.; Cardner, C. R.; Gillespie, R. J.; Westwood, R.; J. Med. Chem. 1991, 34, 2060.
20. Klingsberg, E.; J. Am. Chem. Soc. 1958, 80, 5786.

21. Kerr, N. V.; Ott, D. G.; Hayes, F. N.; J. Am. Chem. Soc. 1960 , $82,186$.

22. Short, F. W.; Long, F. M.; J. Heterocycl. Chem. 1969, 6, 707.

23. Brain, C. T.; Paul, J. M.; Loong, Y.; Oakley, P. J.; Tetrahedron Lett. 1999, 40, 3275.

24. Brain, C. T.; Brunton, S. A.; Synlett 2001, 382.

25. Dabiri, M.; Salehi, P.; Baghbanzadeh, M.; Zolfigol, M. A.; Bahramnejad, M.; Synth. Commun. 2007, 37, 1201.

26. Polshettiwar, V.; Varma, R. S.; Tetrahedron Lett. 2008, 49, 879.

27. Shaterian, H. R.; Ghashang, M.; J. Braz. Chem. Soc. 2008, 19, 1053.

28. Lenardão, E. J.; Silva, M. S.; Mendes, S. R.; Azambuja, F.; Jacob, R. G.; Santos, P. C. S.; Perin, G.; J. Braz. Chem. Soc. 2007, 18, 943.

29. Perin, G.; Jacob, R. G.; Dutra, L. G.; Azambuja, F.; Santos, G. F. F.; Lenardão, E. J.; Tetrahedron Lett. 2006, 47, 935.

30. Mohsenzadeh, F.; Aghapoor, K.; Darabi, H. R.; J. Braz. Chem. Soc. 2007, 18, 297.

31. Sena, V. L. M.; Srivastava, R. M.; de Simone, C. A.; Gonçalves, S. M. C.; Silva, R. O.; Pereira, M. A.; J. Braz. Chem. Soc. 2007, 18,1224 .

32. Moghaddam, F. M.; Bardajee, G. R.; Ismaili, H.; J. Braz. Chem. Soc. 2007, 18, 1024.

33. Heravi, M. M.; Sabaghian, A. J.; Bakhtiari, K.; Ghassemzadeh, M.; J. Braz. Chem. Soc. 2006, 17, 614.

34. Braibante, M. E. F.; Braibante, H. T. S.; Morel, A. F.; Costa, C. C.; Lima, M. G.; J. Braz. Chem. Soc. 2006, 17, 184.

35. Dabiri, M.; Salehi, P.; Baghbanzadeha, M.; Bahramnejad, M.; Tetrahedron Lett. 2006, 47, 6983.

36. Siegrist, A. E.; Maeder, E.; Duennenberger, M.; Swiss Patent 383985, 1959.

37. Neelima; Bhaduri, A. P.; Indian J. Chem. Sect. B 1983, 22, 79.

38. Karnik, A. V.; Kamath, R. G.; Indian J. Chem. Sect. B 1997, $36,803$.

39. Park, Y. D.; Kim, J. J.; Chung, H. A.; Kweon, D. H.; Cho, S. D.; Lee, S. G.; Yoon, Y. J.; Synthesis 2003, 560.

40. Koldobskii, G. I.; Ivanova, S. E.; Russ. J. Gen. Chem. 1994, 64, 1698.

41. Weidinger, H.; Kranz, J.; DE Patent 1067439, 1958.

42. Myznikov, Y. E.; Koldobskii, G. I.; Vasileva, I. N.; Ostrovskii, V. A.; J. Org. Chem. USSR (Engl.Transl.) 1988, 24, 1397.

Received: May 5, 2008 Web Release Date: October 10, 2008 